\title{
Static Voltage Stability Analysis of Power System Using the Combination of P-V Curve and the Modal Analysis
}

\author{
Wang-Qing Mao ${ }^{1, a,}$, Qi Yang ${ }^{2, ~ b}$, Ming-Yu Yang ${ }^{1, c}$, Shuang Zhang ${ }^{3}$, Feng Gao ${ }^{3}$, \\ Yun-ting Song ${ }^{2, d}$
}

${ }^{1}$ School of Electrical and Electronic Engineering, North China Electric Power University, Baoding 071003, China

${ }^{2}$ China Electric Power Research Institute, Bejing 100192, China

${ }^{3}$ Ningxia Electric Power Research Institute, Yinchuan 750002, Ningxia Hui Autonomous Region, China

amaowangqing@163.com, byangqi@epri. sgcc.com.cn, cyangmy1026@hotmail. com, ${ }^{d}$ syt@epri.sgcc.com.cn

Keywords: Voltage Stability, P-V Curve, Modal Analysis, Weak Area

\begin{abstract}
Acorrding to the poor effect of traditional voltage stability method in the actual power network, a new method, combination of the P-V curve and modal analysis, was put forward. First, $\mathrm{Kp}$ (power reserve coefficient) was calculated to measure the power system static voltage stability margin through P-V curve. Then, on the basis of that, the modal method was used to get a detailed analysis. The participation factors under the minimum singular value of each node were calculated to find out the weak area, the key branch and key generators of the power system. These results may guide operators to note the weak areas, timely adjust the power flow, and arrange reactive power compensation and so on after a major disturbance. The study of a large power grid, with the help of the PSD-Vsap, a static voltage stability program, verifies the validity and practicability of the method.
\end{abstract}

\section{Introduction}

Voltage stability refers to the ability to maintain the voltage of power system, that is in power system, every bus voltage in normal or disturbed after a dynamic process can be controlled within its rated voltage tolerance range. The occurrence of the United States and Canada blackout in August 14, 2003, the Moscow blackout in May 25, 2005, the Europe blackout in November 4, 2006 and a series of accidents, caused a wide attention of all countries in the world on the voltage stability $^{[1]}$. In the background of the rapid growth of electricity load, how to ensure the safe and stable operation of the power grid to ensure reliability of power supply has become an urgent problem to be solved. Current methods of voltage stability analysis mainly include the maximum power method ${ }^{[2]}$, sensitivity method ${ }^{[3,4]}$, the eigenvalues and singular value decomposition $\operatorname{method}^{[5,}$ ${ }^{6]}$, the multi-solution of power load method $^{[7]}$, the differences analysis ${ }^{[7]}$ etc. These static analysis methods are mostly applied in theoretical research, but when extended to the real complex system, the effect also need to be verified ${ }^{[8,9]}$.

This paper uses P-V curve and the modal analysis to study the combined application method of power system static voltage stability problem. First calculate the power reserve coefficient Kp from the P-V curve, which measures the power system static voltage stability margin. Then on the basis, apply the modal analysis method, from the perspective of the whole power system, get detailed information relating to the analysis of the weak areas and provide a basis for best location choice to compensate reactive power.

The P-V curve method is an method based on power flow simulation to study the voltage stability of power system ${ }^{[10]}$, it is the effective tool for judging system static voltage stability margin. We use PSD-Vsap static voltage stability analysis program to draw PV curve. The applications of modal analysis method in other areas are more mature, but less on static voltage stability analysis ${ }^{[11,}$ 
12]. Modal analysis method can be used for the analysis of actual system, the actual example analysis shows that, the method is effective and practical.

\section{PV and QV Curves and Voltage Stability Margin}

In the power system, PV and QV curves of load nodes can be used as an effective tool to determine the system static voltage stability margin. P-V or Q-V curve, as shown in Fig.1, the abscissa represents total load $\mathrm{P}$ or $\mathrm{Q}$ of a node, the vertical axis represents the voltage amplitude corresponding to the load. $\mathrm{P}-\mathrm{V}$ and $\mathrm{Q}-\mathrm{V}$ curve inflection point is the critical point of stable operation. The load Pmax corresponding to inflection point is the ultimate load, and the voltage Vcr corresponding to the inflection point is the limit voltage of the stable operation. The difference between the ultimate power and load of the actual operating point, as well as the voltage difference between the limit and actual voltage operating point can be integrated to reflect the static voltage stability margin of the node.

In practical applications, the power reserve coefficient $K p$ and voltage reserve factor $K v$ are often used to reflect the static voltage stability margin of the system .The power reserve coefficient $K p$ and voltage reserve factor $K v$ are defined as Eq.1 and Eq.2:

$$
\begin{aligned}
& K_{p}=\frac{P_{\max }-P}{P} \\
& K_{v}=\frac{V_{o}-V_{c r}}{V_{c r}}
\end{aligned}
$$

In Eq.1 and Eq.2 , Pmax is the limit of active power, $P$ is the active power under normal operation, $V o$ is the normal operating voltage, $V c r$ is the critical operating voltage.

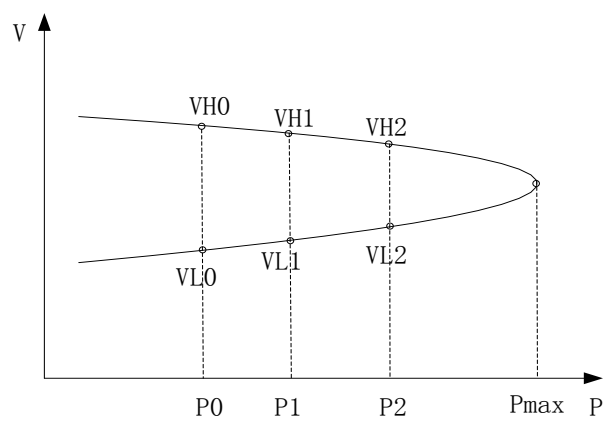

Fig.1 Typical P-V Curve

With linear simulation method, we can quickly get PV and QV curves to calculate the power reserve coefficient $K p$ and voltage reserve coefficient $K v$. Of the two, the system static voltage stability and power reserve factor $K p$ are in good agreement, and when the P-V curve is relatively flat, voltage reserve coefficient $\mathrm{Kv}$ can not well reflect the static voltage stability.So our main method to measure static voltage stability of the system is the $K p$.

\section{Application of Modal Analysis Technology in Voltage Stability Analysis}

Static voltage stability of the system is derived from a PV curve, power system voltage static linear equation can be expressed as: 


$$
\left[\begin{array}{c}
\Delta P \\
\Delta Q
\end{array}\right]=\left[\begin{array}{ll}
J_{P \theta} & J_{P V} \\
J_{Q \theta} & J_{Q V}
\end{array}\right]\left[\begin{array}{c}
\Delta \theta \\
\Delta V
\end{array}\right]=J\left[\begin{array}{c}
\Delta \theta \\
\Delta V
\end{array}\right]
$$

In Eq.3, $\Delta P$ and $\Delta Q$ are bus active power and reactive power incremental change, $\Delta \theta$ is the incremental change of bus voltage angle, $\Delta U$ is the incremental change of bus voltage magnitude, $J$ is the Jacobi matrix of the system. We can get the relationship among the power incremental change,right eigenvector and state variables shown in Eq.4 and Eq.5 after mode method is applied to the power flow equations:

$$
\begin{aligned}
& {\left[\begin{array}{c}
\Delta P \\
\Delta Q
\end{array}\right]=K_{i} U_{i}} \\
& {\left[\begin{array}{c}
\Delta \theta \\
\Delta U
\end{array}\right]=\lambda_{i}^{-1}\left[\begin{array}{l}
\Delta P \\
\Delta Q
\end{array}\right]}
\end{aligned}
$$

In Eq.4 and Eq.5, $\Delta \theta$ and $\Delta U$ are incremental change of voltage angle and magnitude, $\lambda_{i}$ is the convergent Jacobi matrix eigenvalues, $\Delta P$ and $\Delta Q$ are power incremental change.In modal analysis of static voltage stability, the right eigenvectors corresponding to the minimum eigenvalue $u_{m}$ reflect the most sensitive perturbation direction of active and reactive power relative to the minimal model ,when the power perturbation direction consistent with $u_{m}$, the changes of state caused is maximum. At this time, the branches reactive power loss which are more sensitive to changes can be considered a greater impact on the minimum model,these kind of branches are defined as the key branch. In the minimal model, the generator whose reactive power changes in larger unit is defined as the key generator. Besides, according to the contribution degree to the node voltage sensitivity, we can find the load node in strong correlation with minimum singular mode,which is defined as the key node. The key nodes constitute weak area, and often are the best location for reactive power compensation.

\section{Case Analysis}

Take a northwest power grid in China for example, the power grid include $4500 \mathrm{kV}$ substations, $16220 \mathrm{kV}$ substations, and the total installed capacity is 9818MW.The northwest power grid is the load center of a regional power grid. Power reference value is 100MVA.

\section{Static Voltage Stability Margin Analysis of Normal Operation Mode}

Research on the modeling of the power grid, under the high load operation mode in the summer of 2013, large part of static stability indices of main results are shown in Table 1. Table 1 shows the voltage stability index of main load bus: the power reserve coefficient Kp. According to "Guidelines for power system security and stability"published by China Electric Power Research Institute, aiming at different power systems under the normal mode of operation, static stability reserve coefficient is calculated according to the power angle criterion should satisfy $15 \%-20 \%$. From Table 1 : under the high load operation mode in the summer of 2013, power reserve coefficient Kp is above $31 \%$, so there is a larger margin.Gu North regional power reserve coefficient is low, the power reserve is relatively weak. 
Table 1 The Main Static StabilityResults of the $115 \mathrm{kV}$ Nodes

\begin{tabular}{cccc}
\hline Node & $\begin{array}{c}\text { Voltage } \\
\text { Level[kV] }\end{array}$ & Power Limit[p.u.] & Kp [\%] \\
\hline Baogang 52 & 115.0 & 3.488 & 98.39 \\
Gu North11 & 115.0 & 2.495 & 31.64 \\
Hezi 11 & 115.0 & 3.145 & 97.67 \\
Kuang West 11 & 115.0 & 1.607 & 83.53 \\
Shibao 11 & 115.0 & 1.798 & 72.43 \\
Huabian 11 & 115.0 & 1.257 & 96.42 \\
Dou West 11 & 115.0 & 2.058 & 84.88 \\
\hline
\end{tabular}

Fig.2 shows the P-V curves of the Huabian $115 \mathrm{kV}$ bus, under the high load operation mode in the summer of 2013 . The Kp of Huabian $115 \mathrm{kV}$ bus is $96.42 \%$. According to the power reserve coefficient under different operation modes, there is a certain distance between the operation point of the system and the power limit, so the grid has enough static voltage stability margin.

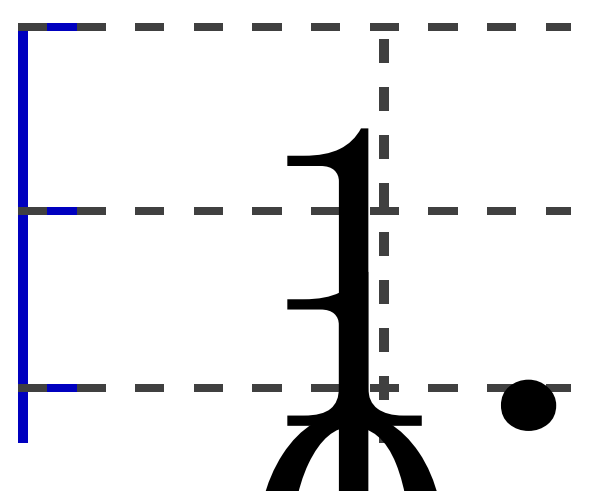

Fig.2 P-V Curve of the Hubian $115 \mathrm{kV}$ Bus

\section{Calculation Results of Modal Analysis}

The major part of related factors calculation results of load bus, transmission lines and generators, under the high load operation mode in the summer of 2013, are shown in Table 2 to 4 .

The buses whose related factor is relatively big belong to voltage stability weak point, and the greater its value, the greater the contribution to the node voltage sensitivity; the greater line participation factor, the more sensitive to reactive power loss; the greater generator participation factor, the greater issued reactive power change.

For the high load operation mode in summer, Barun, Kuang West , Baiyun, Gu North are the voltage stability relatively weak points; Gu North 220-Chunkun 220 lines, Tuyou 220-Daqi 220, Zhao Temple 220-Sha River 220, Baobai 115-Gu North 115, G1 and G2 generator of River West, are the corresponding key branches and key generators.

Table 2 The Main Related Factor Results of Buses

\begin{tabular}{cccc}
\hline Number & Node & Voltage Level[kV] & Related Factor \\
\hline 1 & Barun 12 & 115.0 & 0.551465 \\
2 & Kuang West 11 & 115.0 & 0.503995 \\
3 & Baiyun 11 & 115.0 & 0.501605 \\
4 & Shibao 11 & 115.0 & 0.349154 \\
5 & Guyang 12 & 115.0 & 0.346726 \\
6 & Goden Mount 11 & 115.0 & 0.336117 \\
7 & Baobai T2 & 115.0 & 0.334535 \\
\hline
\end{tabular}


Table 3 The Main Related Factor Results of Transmission Lines

\begin{tabular}{ccccc}
\hline Number & Voltage Level[kV] & Node 1 & Node 2 & Related Factor \\
\hline 1 & 220 & Gu North 21 & Chunkun 21 & 0.132382 \\
2 & 220 & Zhao Temple 21 & Sha River 21 & 0.042807 \\
3 & 220 & Gu North 21 & Red Tower 21 & 0.038627 \\
4 & 220 & Wang Sea 22 & Red Tower 21 & 0.028005 \\
5 & 110 & Barun 11 & Wang Sea12 & 0.013853 \\
6 & 110 & Xingqing T1 & Happiness 11 & 0.009733 \\
7 & 110 & Baobai T1 & Baobai T2 & 0.009688 \\
8 & 110 & Shashi T1 & Sha River11 & 0.007306 \\
\multicolumn{5}{|c}{ Table 4 The Main Related Factor Results of Generators } \\
\hline \multirow{2}{*}{ Number } & Generator & Voltage & \multirow{2}{*}{ Related Factor } & \multirow{2}{*}{ Reactive power reserve[p.u.] } \\
\hline 1 & River West G1 & 20 & 0.3734 & 1.706 \\
2 & River West G2 & 20 & 0.3668 & 1.706 \\
3 & Bao Third G1 & 20 & 0.2506 & 0.504 \\
4 & Daqi G2 & 24 & 0.2283 & 0.504 \\
\hline
\end{tabular}

\section{Analysis of the Computation Result}

According to the static voltage stability margin analysis ,we can effectively evaluate the existing power grid's capability of bearing load growth. The power limit of northern ,southern, western, eastern power grid is about 1.5pu (907MW), 2.0pu (1950MW), 1.9pu (3425MW), 2.2pu (4635MW) respectively. Barun, Kuang West, Baiyun, Gu North are relatively weak point of voltage stability, and they can be choosen as the preferred position for reactive power and voltage compensation.

\section{Conclusions}

This paper presents a new method based on PV curve method combined with modal applications. By studying the results of the actual grid shows that the method is simple and effective. Operating personnel in the normal scheduling and monitoring, should pay attention to the key lines, reduce the load on the key lines and make reasonable adjustments on tide way when a major disturbance occured in the system. Besides,key generators plays a key role for the stability of the system, Operating personnel should consider arranging adequate reserves for them to avoid systems lossing voltage stability.

\section{References}

[1] Hong Yu: Relay,Vol. 35(2007), p. 55-60, in Chinese.

[2] Custem: IEEE Transactions on Power Systems, Vol. 6(1991), p. 145-156.

[3] Feifei Dong, Ailing Liu , Chaoyang Xiong : Shaanxi Electric Power, in Chinese, Vol.5(2013), p. 1-4, in Chinese.

[4] Jung Yun, Xianzhong Duan, Yangzan He: Power System Technology, Vol. 21(1997), p. 7-10, in Chinese.

[5] Zhihong Feng, Qu Liu, Xiyin Ni: Proceedings of the CSEE, Vol. 12(1992), p. 10-19, in Chinese.

[6] Lingzhi Lin, Bo Zhang, Sen Zou: Journal of Shangdong University of Technology,Vol. 26(1996), p. 148-154, in Chinese. 
[7] Hua Yin, Jianyong Ding: Northeast Electric Power Technology, Vol. 12(2003), p. 8-10, in Chinese.

[8] Xiuping Wu, Jun Liu: North China Electric Power, Vol. 23(2007), p. 4-6 in Chinese.

[9] Sheng Xiao,Jianhua Zhang,Shifan Guo: Power System and Clean Energy, Vol. 26(2010), p. 10-13, in Chinese.

[10] Weisheng Wang: Power system voltage stability( China Power Press, China, 2002).

[11]Quan Li, Jianwen Ren, Wenping Hu: Shaanxi Electric Power, Vol. 13(2011), p. 28-32, in Chinese.

[12]Weichao Wang,Wanjun Wang, Jianxing Li: Shaanxi Electric Power,Vol. 09(2006), p. 8-11, in Chinese. 